\title{
mTOR complex 2 phosphorylates IMP1 cotranslationally to promote IGF2 production and the proliferation of mouse embryonic fibroblasts
}

\author{
Ning Dai, ${ }^{1,2,3}$ Jan Christiansen, ${ }^{4}$ Finn C. Nielsen, ${ }^{5}$ and Joseph Avruch ${ }^{1,2,3,6}$ \\ ${ }^{1}$ Department of Molecular Biology, ${ }^{2}$ Diabetes Unit, Medical Services, Massachusetts General Hospital, Boston, Massachusetts \\ 02114, USA; ${ }^{3}$ Department of Medicine, Harvard Medical School, Boston, Massachusetts 02115, USA; ${ }^{4}$ Department of Biology, \\ ${ }^{5}$ Department of Clinical Biochemistry, Rigshospitalet, University of Copenhagen, 2100 Copenhagen, Denmark
}

\begin{abstract}
Lack of IGF2 in mice results in diminished embryonic growth due to diminished cell proliferation. Here we show that mouse embryonic fibroblasts lacking the RNA-binding protein IMP1 (IGF2 mRNA-binding protein 1) have defective splicing and translation of IGF2 mRNAs, markedly reduced IGF2 polypeptide production, and diminished proliferation. The proliferation of the IMP1-null fibroblasts can be restored to wild-type levels by IGF2 in vitro or by re-expression of IMP1, which corrects the defects in IGF2 RNA splicing and translation. The ability of IMP1 to correct these defects is dependent on IMP1 phosphorylation at Ser181, which is catalyzed cotranslationally by mTOR complex 2 (mTORC2). Phosphorylation strongly enhances IMP1 binding to the IGF2-leader $35^{\prime}$ untranslated region, which is absolutely required to enable IGF2-leader 3 mRNA translational initiation by internal ribosomal entry. These findings uncover a new mechanism by which mTOR regulates organismal growth by promoting IGF2 production in the mouse embryo through mTORC2-catalyzed cotranslational IMP1/IMP3 phosphorylation. Inasmuch as TORC2 is activated by association with ribosomes, the present results indicate that mTORC2-catalyzed cotranslational protein phosphorylation is a core function of this complex.
\end{abstract}

[Keywords: mTOR complex 2; IMP1; cotranslational phosphorylation; mRNA translation; IGF2; embryonic growth] Supplemental material is available for this article.

Received October 29, 2012; revised version accepted December 26, 2012.

Organismal size is determined by genetic and environmental (especially nutritional) factors. Two sets of conserved genes important to organismal size are those of the mTOR and insulin-IGF pathways (Efstratiadis 1998; Hietakangas and Cohen 2009; Laplante and Sabatini 2012). The phylogenetically older mTOR kinase is a dominant regulator of cell size in single-cell eukaryotes, responding to nutritional and stressful stimuli to control mRNA translation. In metazoans, mTOR continues to regulate cell size in response to nutrients but is coregulated by the insulin-IGF pathway, which coordinates overall organismal growth. Here we identify a new mechanism by which mTOR regulates organismal growth acting upstream of IGF2.

During embryogenesis in mice, IGF1 and IGF2, acting primarily through the IGF1 receptor, are the primary determinants of birth size; inactivation of either IGF reduces size at birth to $60 \%$ of wild type due to reduced cell proliferation, and their combined deficiency reduces

${ }^{6}$ Corresponding author

E-mail avruch@molbio.mgh.harvard.edu

Article is online at http://www.genesdev.org/cgi/doi/10.1101/gad.209130.112. birth size in an additive fashion. Although IGF2 expression is detectable at the two-cell stage and is strong in extraembryonic tissues as early as embryonic day 4.5 (E4.5), expression in the embryo is not evident until E7.5, increasing rapidly thereafter (Lee et al. 1990). Nevertheless, embryos lacking IGF2 or the IGF1 receptor do not show growth retardation until E11 (DeChiara et al. 1990; Efstratiadis 1998). IGF2 RNAs are expressed by the paternal allele from at least four promoters (P0-P3), each transcript differing from the others only in their 5' untranslated regions (UTRs) (Sussenbach 1989; Constância et al. 2002); the dominant mRNA species in the embryo are those expressed from P2, encoding a 1170-nucleotide (nt) 5' UTR (henceforth called leader 3 or L3), and P3, which encodes a 100-nt 5' UTR (henceforth called leader 4 or L4) (Newell et al. 1994). The translation of the IGF2 mRNAs during mouse embryogenesis is subject to regulation (Newell et al. 1994), mediated at least in part by the IGF2 mRNA-binding proteins or IMPs (IMP1-3) (Nielsen et al. 1999; Hansen et al. 2004).

The IMPs were identified through their ability to bind to the IGF2-L3 5' UTR (Nielsen et al. 1999) and were 
shown to also bind to a site on the $3^{\prime}$ UTR shared by all IGF2 mRNAs. IMP1-3 are homologous $60-70-\mathrm{kDa}$ proteins that contain two RNA recognition motif (RRM) domains followed by four hnRNP $\mathrm{K}$ homology $(\mathrm{KH})$ domains (Nielsen et al. 2001; Yisraeli 2005). The IMPs are perhaps best known for their role in RNA transport, as exemplified by the chicken IMP1 ortholog Zipcodebinding protein-1 (ZBP-1), which binds to a 54-nt segment in the $3^{\prime}$ UTR of $\beta$-actin mRNA and suppresses its translation while mediating its translocation to the cell periphery (e.g., of fibroblasts); there, cSrc-catalyzed phosphorylation of ZBP-1[Tyr396], a site located between the second and third $\mathrm{KH}$ domains, causes release of the $\beta$-actin mRNA, enabling its localized translation (Hüttelmaier et al. 2005). In addition to their ability to regulate mRNA transport, the IMPs have also been implicated in RNA stabilization against endonuclease attack and in both positive and negative regulation of translational initiation (Nielsen et al. 2001; Yisraeli 2005).

In mouse embryos, expression of IMP1 is evident at E10.5, and mRNA for all three IMPs increases markedly at E12.5 (Nielsen et al. 1999), coinciding with the onset of IGF2 action in the embryo; like IGF2, IMP1 and IMP3 expression is largely extinguished after birth, whereas IMP2 expression persists in the adult. In mouse embryos lacking IMP1, the overall abundance of IGF2-L3 and IGF2-L4 mRNAs at E12.5 is unaltered from wild type, but their polysomal abundance is reduced. The weight of IMP1-null mouse embryos becomes lower than wild type after E14.5 due to reduced cell proliferation, and the birth weight of IMP1-null mice is $\sim 75 \%-80 \%$ of wild type (Hansen et al. 2004). Thus, IMP1 is an important determinant of embryonic growth in mice, an action attributable in part to IMP1 regulation of IGF2 mRNA translation.

In the rhabdomyosarcoma cell line $\mathrm{RD}$, the translation of IGF2-L3 mRNA, which occurs by cap-independent internal ribosome entry, is selectively inhibited by rapamycin (Nielsen et al. 1995). This action of rapamycin is mediated by inhibition of mTOR complex 1 (mTORC1)catalyzed dual phosphorylation of IMP-2[Ser162/Ser164]; loss of this dual phosphorylation causes dissociation of IMP2 from the L3 5' UTR, accompanied by inhibition of IGF2-L3 mRNA polysomal association (Dai et al. 2011). This observation led us to inquire whether IMP1 and IMP-3 are substrates for and regulated by mTOR-catalyzed phosphorylation. IMP1 and IMP3 lack phosphorylatable residues at the sites corresponding exactly to IMP2[Ser162/164], which are adjacent Ser-Pro residues situated between the second RRM domain and the first $\mathrm{KH}$ domain. Nevertheless, IMP1 and IMP3 do each encode a single Ser-Pro residue between RRM2 and KH1 at Ser181 and Ser183, respectively, and phosphorylation of IMP1[Ser181] has been observed (Huttlin et al. 2010; summarized at http://www.phosphosite.org). Here we confirm the phosphorylation of IMP1 at Ser181 and identify phosphorylation of IMP3[Ser183]. In contrast to the dual phosphorylation of IMP2[Ser162/Ser164], which is catalyzed by mTORC1 in an insulin-stimulated post-translational manner, IMP1[Ser181] phosphoryla- tion is a cotranslational modification catalyzed by mTORC2. Loss of this cotranslational phosphorylation greatly impairs IMP1's ability to enable proper splicing and translation of IGF2 RNA and greatly reduces IGF2 production by fibroblasts isolated from E13.5 IMP1-null mouse embryos. The proliferative rate of IMP1-null mouse embryonic fibroblasts (MEFs) in vitro is substantially reduced but can be restored to wild-type levels by IGF2 or re-expression of IMP1 but not by IMP1[Ser181Ala]. Thus, mTORC2, through its cotranslational phosphorylation of the nascent IMP1 (and IMP3) proteins, is required for optimal IGF2 production by and for growth of the mouse embryo.

\section{Results}

\section{IMP1 and IMP3 are phosphorylated by mTOR}

IMP1 and IMP3, immunoprecipitated from rapidly growing RD rhabdomyosarcoma cells (Nielsen et al. 1995), were subjected to tryptic digestion followed by liquid chromatography tandem mass spectrometry (LC-MS/ MS); a single site of phosphorylation was identified in each protein: IMP1[Ser181] and IMP3[Ser183]. To facilitate detection of these phosphorylations in intact cells, polyclonal phospho-specific antibodies were generated by immunization of rabbits with the corresponding synthetic phosphopeptide coupled to Keyhole Limpet Hemocyanin (KLH) (IMP1[Ser181P]: CysQPRQGS[PO4]PVAAGamide; IMP3[Ser183P]: CysSSRQGS[PO4]PGSVSamide). After purification, each antibody exhibited immunoblot reactivity against recombinant wild-type IMP1 or IMP3 but not against IMP1[Ser181Ala] (Fig. 1A, top) or IMP3[Ser183Ala] (Fig. 1A, bottom), respectively. In view of the homologous nature of the sites of IMP1 and IMP3 phosphorylation, we focused the following studies on IMP1.

To screen for protein kinases capable of phosphorylating the IMP1 Ser-Pro sites, we treated RD cells with relatively high concentrations of several agents capable of inhibiting a variety of proline-directed kinases, including MAPKs, cdks, GSK3, and mTOR (Davies et al. 2000; Bain et al. 2003; Thoreen et al. 2009). Although rapamycin (0.2 $\mu \mathrm{M}, 2 \mathrm{~h})$ was entirely without effect (data not shown), Torin1 gave significant, albeit partial reduction of IMP1[Ser181] (Fig. 1B). The phosphorylation of IMP1[Ser181] remained detectable at 0.4-0.6 $\mu \mathrm{M}$ Torin 1, whereas the phosphorylation of S6K[T389] and the dual phosphorylations of 4E-BP[Thr37/46] and IMP2[Ser162/164] were largely eliminated at $0.2 \mu \mathrm{M}$ Torin1 (Fig. 1C). Despite the relative resistance of IMP1[Ser181P] to Torin1, shRNAinduced depletion of $\mathrm{mTOR}$ is accompanied by a substantial decrease in IMP1[Ser181P] (Fig. 1D).

\section{IMP1 phosphorylation is cotranslational and is catalyzed by mTORC2}

Seeking to understand the limited inhibitory effect of Torin1 on IMP1[Ser181] phosphorylation, we observed that IMP1[Ser181P] is also quite resistant to dephosphorylation in lysates of RD cells or MEFs; however, addition 


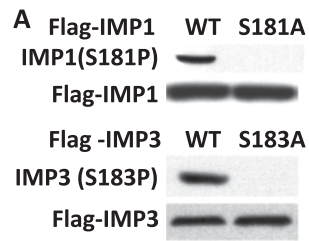

C

[Torin1 nM], 60': 0200400600

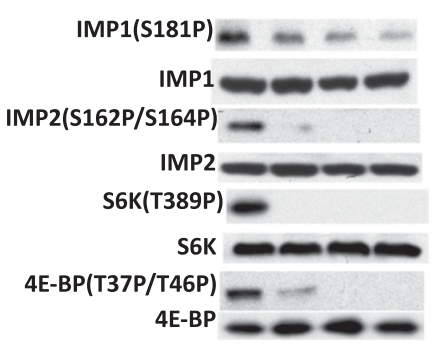

B

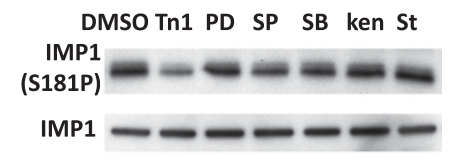

D

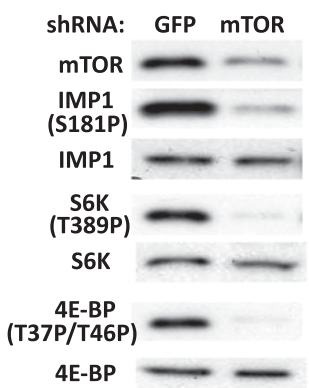

Figure 1. IMP1(Ser181) phosphorylation is mTOR-dependent. (A) The phospho-specificity of the anti-IMP1(Ser181-P) and IMP3(Ser183-P) antibodies. Flag-IMP1 and Flag-IMP1(Ser181Ala) (top) or IMP3 wild type (WT) and IMP3(S183A) (bottom) were expressed transiently in RD cells; extracts were blotted for Flag and for IMP1(Ser181-P) or IMP3(Ser183P). (B) IMP1(Ser181) phosphorylation in RD cells is partially inhibited by Torin 1 but not by Staurosporine or inhibitors of proline-directed protein kinases. RD cells were treated for $1 \mathrm{~h}$ with Torin $1(200 \mathrm{nM})$, PD $184352(25 \mu \mathrm{M})$, SP $600125(50 \mu \mathrm{M})$, SB $2023580(25 \mu \mathrm{M})$, Kenpaullone $(20 \mu \mathrm{M})$, Staurosporine $(10 \mu \mathrm{M})$, and DMSO. Extracts were blotted for IMP1 and IMP1(Ser181-P). (C) IMP1 S181 phosphorylation is resistant to Torin1-induced dephosphorylation. RD cells were treated with Torin 1 at the doses indicated for $1 \mathrm{~h}$; extracts were immunoblotted for IMP1, IMP2, S6K1, 4E-BP, and the phosphorylation sites on each of those proteins as indicated. $(D)$ Depletion of mTOR from RD cells reduces the phosphorylation of IMP1(Ser181) and other mTOR substrates.

of RNase A enables rapid and complete IMP1[Ser181P] dephosphorylation without altering the abundance of the IMP1 polypeptide (Fig. 2A). The RNase-induced dephosphorylation can be fully inhibited by calyculin (Fig. 2B, left) or $\mathrm{NaF}$ (Fig. 2B, right), entirely distinct potent inhibitors of protein (Ser/Thr) phosphatases (Favre et al. 1997). Thus, RNA binding appears to protect IMP1[Ser181P] from protein phosphatase action. This suggests that the partial loss of IMP1[Ser181] phosphorylation in the presence of Torin 1 may reflect slow turnover of some IMP1-RNA complexes, thereby limiting susceptibility of those RNA-associated IMP1 polypeptides to [Ser181P] dephosphorylation despite inhibition of mTOR kinase.

RNase treatment of cell extracts greatly reduces the coprecipitation of endogenous IMP1 with IMP2 and IMP3 (Fig. 2C), indicating that IMP-IMP heteroassociation is mediated largely by their ability to bind shared RNAs. RNase pretreatment also promotes the association of IMP1 with mTOR (as seen previously with IMP2) (Dai et al. 2011); however, the ability of RNase treatment to promote the IMP-mTOR association is prevented by inclusion of the phosphatase inhibitors $\mathrm{NaF}$ or calyculin. (Fig. 2B). Thus, dephospho-IMPs bind preferentially to mTOR; moreover, inasmuch as RNase abolishes IMP hetero-oligomers, it follows that dephospho-IMP1, IMP2 (Dai et al. 2011), and IMP3 (data not shown) each binds to mTOR directly; i.e., independently of the other IMPs. After RNase treatment of lysates, endogenous IMP1 is observed to coprecipitate selectively with the mTOR fragment [1-670] (Fig. 2D). IMP2 was shown previously to bind mTOR without the need for RNase pretreatment and selectively to mTOR[1265-1967] (Dai et al. 2011); RNase A treatment uncovered the ability of IMP2 to bind mTOR[1-670] as well (Fig. 2D).

We next examined the regulation of IMP phosphorylation in intact cells (Fig. 3A). In 293E cells, overnight removal of serum causes the elimination of S6K1[Thr389] phosphorylation and a marked reduction in 4E-BP[Thr37/ 46] dual phosphorylation; in contrast, IMP1[Ser181] phosphorylation is modestly reduced. Acute addition of a saturating concentration of insulin to serum-deprived 293E cells strongly stimulates S6K1[Thr389] phosphorylation and increases 4E-BP[Thr37/46] substantially but causes little or no increase in IMP1[Ser181P]. Withdrawal of extracellular AA from serum-deprived 293 cells abolishes S6K[Thr389] phosphorylation and 4E-BP[Thr37/46] dual phosphorylation within $1 \mathrm{~h}$, whereas IMP1[Ser181] phosphorylation is not detectably altered at this time. Thus, there appears to be minimal rapid post-translational regulation of IMP1[Ser181] phosphorylation, behavior that suggests that this phosphorylation may occur cotranslationally.

To evaluate more directly whether IMP1[Ser181] phosphorylation occurs cotranslationally, we examined the ability of mTOR to phosphorylate IMP1[Ser181] during translation of IMP1 mRNA in vitro in a reticulocyte lysate. Addition of recombinant wild-type mTOR but not mutant inactive $\mathrm{mTOR}[\mathrm{NK}]$ to rabbit reticulocyte lysates actively translating IMP1 mRNA results in a considerable phosphorylation of the newly synthesized IMP1 polypeptide at Ser181, which is strongly inhibited by Torin 1 (Fig. 3B, lanes 5-7). If, however, IMP1 mRNA is translated to a similar extent in the absence of mTOR, followed by the addition of puromycin to terminate translation and displace nascent polypeptides from the ribosome (Pestka 1971), mTOR added subsequently catalyzes little or no phosphorylation of the newly synthesized, ribosome-free IMP1 (Fig. 3B, lanes 2-4). Recent work has identified the association of mTORC2 with the ribosome and established the ability of mTORC2 to catalyze cotranslational phosphorylation of the "turn" motif of Akt[Ser450] (Oh et al. 2010). In confirmation of this, we found that immunoprecipitation of ribosomes from CHAPS $(0.25 \%)$ extracts of rapidly growing MEFs is accompanied by coprecipitation of $\mathrm{mTOR}$, rictor, and raptor (Fig. 3C). Moreover, after a 10-min treatment of RD cells with puromycin, immunoprecipitates of puromycin prepared from extracts of the puromycin-treated RD cells contain IMP1 phosphorylated at [Ser181] (Fig. 3D), which can only have occurred cotranslationally, and this phosphorylation is 


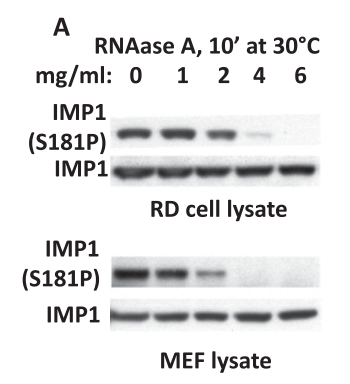

C

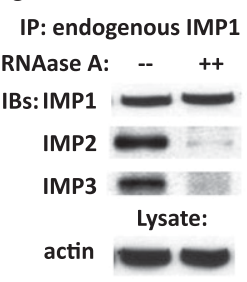

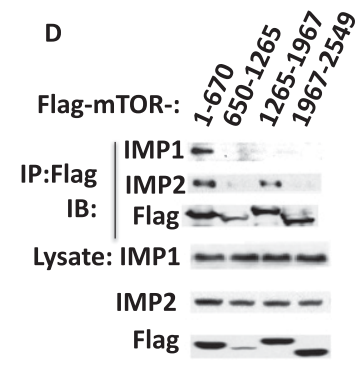

Figure 2. IMP1(Ser181) dephosphorylation enables IMP1 binding to mTOR and is opposed by RNA. $(A)$ RNA protects IMP1(S181P) from dephosphorylation. Extracts of RD cells (top two panels) and MEF cells (bottom two panels) were treated with increasing amounts of RNase A for $10 \mathrm{~min}$ at $30^{\circ} \mathrm{C}$, and the phosphorylation of IMP1 was examined. (B) Dephosphorylation of IMP1(Ser181) promotes IMP1 binding to mTOR. Extracts of $\mathrm{RD}$ cells were incubated for $10 \mathrm{~min}$ at $30^{\circ} \mathrm{C}$ with RNaseA (aseA, $7 \mathrm{mg} / \mathrm{mL}$ ) or RNase inhibitor $(1 \mathrm{U} / \mu \mathrm{L})$. Calyculin $\mathrm{A}(1 \mu \mathrm{M})$ or $\mathrm{NaF}(0.1 \mathrm{M})$ were present as shown. The RD extracts (top two panels) were blotted for IMP1 and IMP1(Ser181-P), and IMP1 immunoprecipitates (bottom two panels) were blotted for IMP1 and mTOR. (C) RNase largely eliminates IMP-IMP heterodimerization. RD cell lysate was treated with $7 \mathrm{mg} / \mathrm{mL}$ RNase A for $10 \mathrm{~min}$ at $30^{\circ} \mathrm{C}$. An IMP1 immunoprecipitate was blotted for IMP1, IMP2, and IMP3. (D) IMP1 and IMP2 binding to mTOR fragments. Flag-mTOR fragments were transiently expressed in $293 \mathrm{~T}$ cells, and extracts were treated with RNase as in C. Flag immunoprecipitations were blotted for endogenous IMP1 and IMP2. inhibited by Torin 1 . Together, these results provide strong evidence that mTOR-catalyzed IMP1 phosphorylation occurs cotranslationally.
To define which mTORC mediates the phosphorylation of the various IMP sites, we examined the impact of shRNA-induced depletion of raptor and rictor from

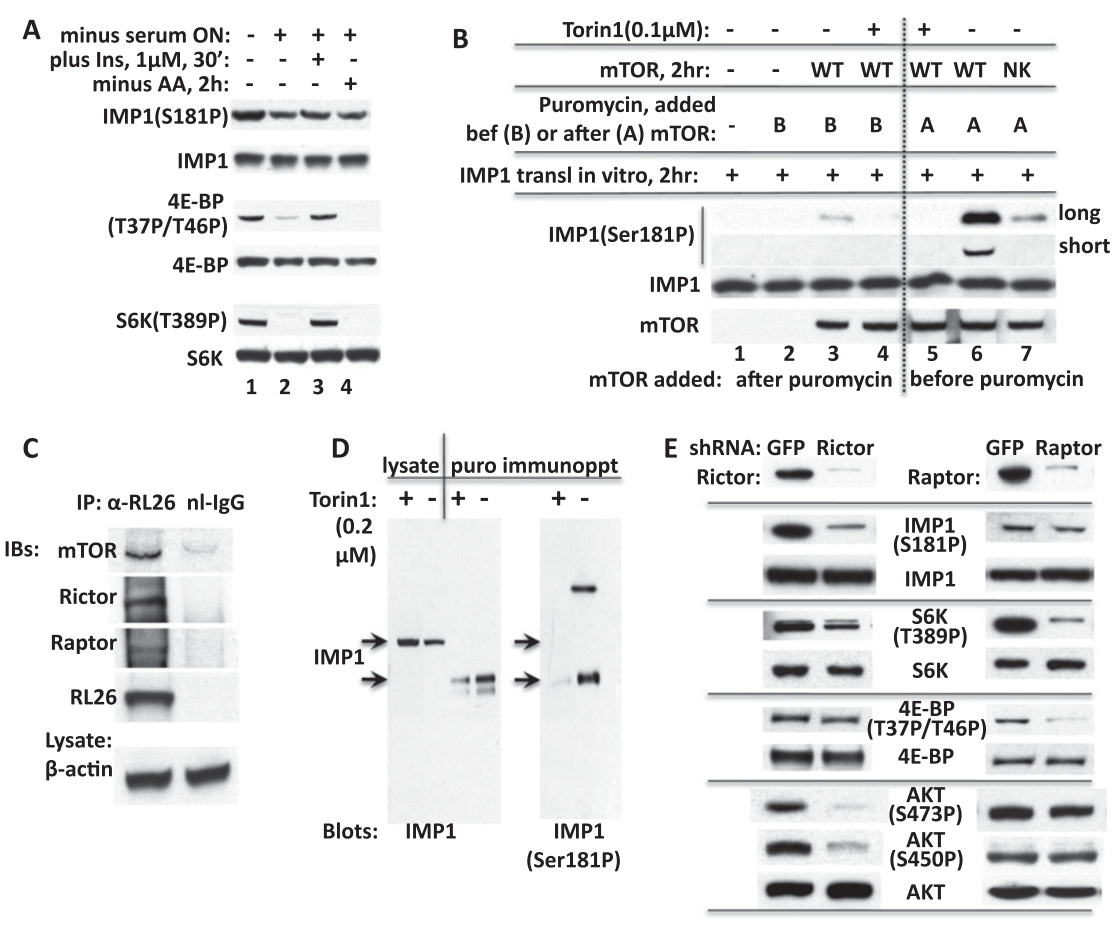

Figure 3. IMP1(Ser181) is phosphorylated cotranslationally by mTORC2. (A) IMP1(Ser181) phosphorylation is unaltered by insulin or amino acid withdrawal. 293E cells were either serum replete (lane 1) or deprived of serum overnight (lanes 2-4). The serum-deprived cells were exposed to insulin $(1 \mu \mathrm{M}, 30 \mathrm{~min}$; lane 3$)$ or placed in DPBS ( $2 \mathrm{~h}$; lane 4). Extracts were blotted for IMP1, S6K1, 4E-BP, and the phosphorylation sites on each as indicated. (B) IMP1 is phosphorylated by mTOR in vitro during, but not after, IMP1 translation. IMP1 mRNA was translated in a rabbit reticulocyte lysate for $2 \mathrm{~h}$. In lanes 5-7, Flag-mTOR (wild type [WT], lanes 5,6; NK, a kinasedead mutant, lane 7) purified from Tritonextracted 293T cells was present during the translation, which was terminated by addition of puromycin, followed immediately by SDS-PAGE sample buffer. In lanes 3 and 4, IMP1 RNA translation proceeded for $2 \mathrm{~h}$ in the absence of added mTOR and was terminated by addition of puromycin. Wild-type Flag-mTOR was then added, and incubation was continued for $2 \mathrm{~h}$ followed by addition of SDS-PAGE sample buffer. The lysate samples were blotted for IMP1,
Rapidly growing MEFs were extracted at $30 \%$ IMP1(Ser181-P), and Flag-mTOR. (C) mTOR and rictor coprecipitate with ribosomes. Rapidly growing MEFs were extracted at 30\%
confluence in buffer containing 0.25\% CHAPS. Immunoprecipitates prepared with rabbit anti-RPL26 (Sigma, R0780) or nl rabbit IgG were subjected to SDS-PAGE and immunoblot as indicated. $(D)$ Nascent IMP1 polypeptides covalently bound to puromycin exhibit Torin1inhibitable Ser181 phosphorylation. Twenty minutes after addition of Torin 1 ( $200 \mathrm{nM})$ to rapidly growing RD cells, puromycin was added at $10 \mathrm{ug} / \mathrm{mL}$, and the cells were extracted $10 \mathrm{~min}$ later. The extracts were blotted for IMP1, and puromycin immunoprecipitates prepared as described by Schmidt et al. (2009) (KeraFast) were separated by SDS-PAGE blotted for IMP1 and IMP1(Ser181-P). (E) Depletion of Rictor but not Raptor from RD cells reduces IMP1(Ser181) phosphorylation. 
rapidly growing $\mathrm{RD}$ cells on the occupancy of relevant phosphorylation sites (Fig. 3E). It is clear that although raptor depletion diminishes the phosphorylation of S6K1[Thr389] and 4E-BP[37/46], it has little effect on the IMP1 phosphorylation, whereas rictor depletion results in the preferential loss in the phosphorylation of IMP1[Ser181] and Akt[Ser450] as well as of Akt[Ser473] (the latter a post-translational phosphorylation). The requirement for rictor led us to inquire whether IMP1 binds rictor (Supplemental Fig. S1). Interestingly, although mature full-length IMP1 is unable to bind mTOR unless it has undergone RNase-enabled dephosphorylation (Fig. 2B), IMP1 does coprecipitate rictor from Triton extracts (wherein $\mathrm{mTOR}$ and rictor are fully dissociated) without prior RNase A and dephosphorylation (Supplemental Fig. S1A). The ability of endogenous IMP1 to bind independently to mTOR and rictor is also readily demonstrated with recombinant TORC components (Supplemental Fig. $\mathrm{S} 1 \mathrm{~B}, \mathrm{C})$. It is likely that the endogenous rictor coprecipitating with IMP1 from the Triton extract (Supplemental Fig. S1A) did reside in $\mathrm{mTORC2}$, inasmuch as precipitation of IMP1 from $0.25 \%$ CHAPS extracts (wherein mTORC2 remains intact) does coprecipitate mTOR and Sin 1 . Thus, IMP1 can bind independently to both mTOR and rictor; only the former requires IMP1 dephosphorylation at Ser181. How the nascent IMP1 polypeptide interacts with ribosome-associated mTORC2 remains to be more fully defined.

In summary, nascent IMP1 binds to mTOR in mTORC2 and is phosphorylated by mTOR cotranslationally at Ser181; this phosphorylation causes the release of IMP1 from mTOR. The subsequent binding of mRNAs to IMP1 protects Ser181P from cellular protein phosphatases.

\section{mTORC2 phosphorylation of IMP1 is required for IMP1 regulation of IGF2 RNA splicing and translation}

We next sought to determine the functional significance of IMP1[Ser181] phosphorylation, primarily through the use of site-specific IMP1 mutants. This approach was facilitated by the availability of MEFs derived from IMP1null mouse embryos (Hansen et al. 2004). The expression of IMP2 and IMP3 polypeptides is very similar in IMP1null MEFs and MEFs from wild-type littermates (Fig. 4A, top). Nevertheless, the IMP1-null MEFs proliferate at $\sim 50 \%$ the rate of wild-type MEFs (Fig. 4A, bottom), a defect remarkably similar to the overall size deficit of

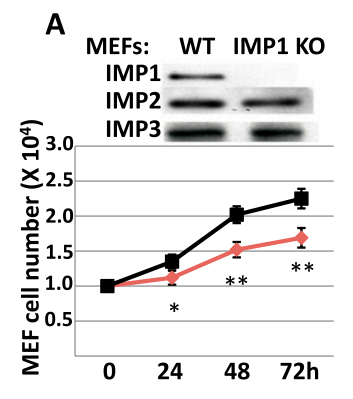

B

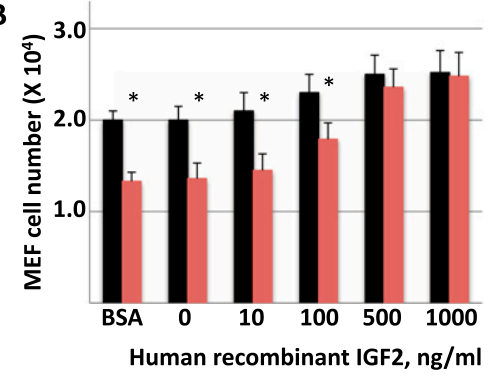

$\mathrm{C}_{\text {DNA }}$

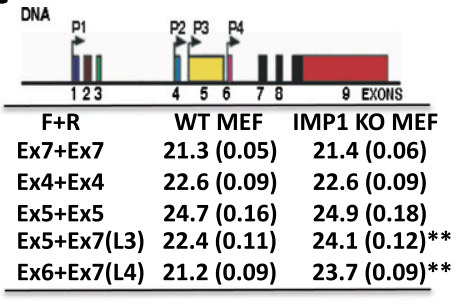

D

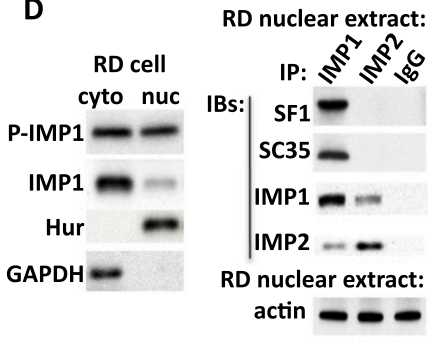

E

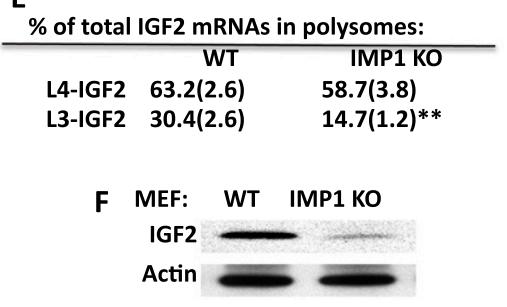

Figure 4. IMP1-null MEFs exhibit markedly reduced IGF2 production due to defective splicing and translation of IGF2 RNA and reduced proliferation that is restored by IGF2. (A) IMP1-null MEFs are hypoproliferative. Identical numbers $\left(10^{4}\right)$ of wild-type or IMP1null MEFs were plated in triplicate at day 0 , and the cell number was counted at 24,48 , and $72 \mathrm{~h}$ thereafter. Results of three experiments, $\pm \mathrm{SD} ;\left(^{\star}\right) P<0.05 ;\left(^{\star \star}\right) P<0.01$ versus wild type. The inset shows blots of cell extracts for IMP1, IMP2, and IMP3. (B) The reduced proliferation of IMP1-null MEFs is restored to wild-type levels by exogenous IGF2. Identical numbers $\left(10^{4}\right)$ of wild-type (black) or IMP1-null (red) MEFs were plated in triplicate at day 0 in the presence of BSA $(1 \mu \mathrm{g} / \mathrm{mL})$ or human recombinant IGF2 at the concentrations indicated. After $48 \mathrm{~h}$, cells were harvested and counted. Three experiments, $\pm \mathrm{SD}_{;}\left(^{\star}\right) P<0.05$. $(C)$ Defective IGF2 RNA splicing in IMP1-null MEFs. The cartoon depicts the exon-intron structure of the IGF2 gene. The Ct values ( \pm SD) from qPCR assays of total RNA for the abundance of specific IGF2 sequences from within exons 7, 4, and 5 and for the spliced L3-IGF2 (Ex5 + Ex7) and L4-IGF2 (Ex6 + Ex7) mRNAs. $\left(^{\star \star}\right) P<0.01$ versus wild type. Exon 7 encodes coding sequences, exon 4 encodes the 5' UTR L2, and exon 5 encodes the 5' UTR L3. (D) Nuclear IMP1 but not IMP2 coprecipitates RNA splicing factors SF1 and SC35 from RD cell nuclear extract. (E) Diminished polysomal association of L3-IGF2 mRNA in IMP1-null MEFs. The percentage ( \pm SD) of total L4-IGF2 mRNA and L3-IGF2 mRNA in the polysomal fractions of sucrose density gradients from extracts of wild-type and IMP1-null MEFs. $\left(^{\star \star}\right) P<$ 0.01 versus wild type. (F) Immunoblot of extracts of wild-type and IMP1-null MEFs for IGF2 and actin. 
the IMP1-null mice at birth (Hansen et al. 2004). The slower proliferation of the IMP1-null MEFs can be brought to wild-type levels by addition of IGF2 (Fig. 4B), suggesting that deficient IGF2 expression underlies their defective proliferation. We therefore compared IGF2 mRNA and protein levels in the wild-type and IMP1-null MEFs. Using quantitative PCR (qPCR), we found that steady-state levels of IGF2 mRNA, as estimated by levels of mRNAs encoding individual exons, was not significantly different in the wild-type and IMP1-null MEFs (Fig. $4 \mathrm{C}$, top three rows); thus, although the impact of IMP1 phosphorylation on IGF2 gene transcription and IGF2 RNA degradation are not known, IMP1 deficiency does not appear to alter the balance of these processes. In contrast, PCR products reflecting the spliced mRNAs corresponding to the L3-IGF2 and L4-IGF2 mRNAs were both substantially diminished in the IMP1-null MEFs by 1.7 cycles for L3 and 2.5 cycles for L4 (Fig. 4C, bottom two rows). Consistent with the participation of IMP1 in IGF2 mRNA splicing, a portion of IMP1 resides in the nucleus, and IMP1 immunoprecipitates from RD cell nuclear extracts contain the splicing factors SF1 and SC3 (Fig. 4D).

In regard to the effect of IMP1 deficiency on IGF2 mRNA translation, the relative abundance of the L4mRNA on polysomes, expressed as a fraction of total L4IGF2 mRNA, is reduced by $10 \%-30 \%$ compared with wild type; however, the fraction of L3-IGF2 mRNA associated with polysomes is reduced from $30 \%$ in the wild-type MEFs to $14 \%$ in the IMP2-null MEFs (Fig. 4E). In addition, the amount of IGF2 polypeptide extracted from the IMP1-null MEFs is markedly diminished as compared with the wild type (Fig. 4F). Thus, IMP1 deficiency reduces the abundance of mature, spliced L3IGF2 and L4-IGF2 mRNAs and inhibits selectively the translation of the L3-IGF2 mRNA, defects that together result in a marked reduction in IGF2 polypeptide production and impaired proliferation.

To determine the effect of IMP1[Ser181] phosphorylation on the abundance and translation of L3-IGF2 and L4IGF2 mRNAs, we generated IMP1-null MEFs that express Tet-inducible versions of IMP1 wild type, IMP1[Ser181Ala], and IMP1[Ser181Asp]. Twenty-four hours after induction of expression of these IMP1 variants to comparable levels (Fig. 5A, top), the abundance and polysome association of L3-IGF2 and L4-IGF2 mRNA was examined. Expression of IMP1 wild type or [Ser181Asp] restored fully the abundance of the mature L3-IGF2 and L4-IGF2 mRNAs to levels comparable with those in wild-type MEFs, whereas IMP1[Ser181Ala] increased the abundance of these mRNAs with approximately one-third the efficacy of the other IMP1 variants (Fig. 5A, bottom). Inasmuch as IMP1 phosphorylation is dependent on rictor, we examined the effect of rictor depletion of the relative ability of IMP1 wild type and IMP1(Ser181Asp) to rescue the abundance of mature L3-IGF2 and L4-IGF2 mRNAs (Fig. 5B). Depletion of rictor was accomplished by infection of the stably transformed MEFs with lentiviral-encoded shRNA, and $2 \mathrm{~d}$ later, the expression of the IMP1 variants was induced with doxycycline; depletion of rictor had little impact on the level of IMP1 polypeptide $24 \mathrm{~h}$ later, but significantly reduced IMP1(Ser181) phosphorylation. Concomitantly, the ability of IMP1 wild type to restore the abundance of L3-IGF2 and L4-IGF2 mRNAs was substantially impaired in the rictor-depleted cells. In contrast, the ability of IMP1(Ser181Asp) to restore L3-IGF2 and L4-IGF2 mRNA expression is completely unaffected by rictor depletion. This provides strong support for the importance of Ser181 phosphorylation in the ability of IMP1 to promote IGF2 RNA splicing.

In regard to the effects of these IMP1 variants on L3IGF2 and L4-IGF2 mRNA polysomal association (Fig. 5C), IMP1 wild type and IMP1[Ser181Asp] modestly enhance the polysomal association of L4-IGF2 mRNA and increase L3-IGF2 mRNA polysomal abundance from 14\% to $\sim 32 \%$ of total, to the levels observed in wild-type MEFs; in contrast, only $16 \%$ of L3-IGF2 mRNA was polysomeassociated in the cells expressing IMP1[Ser181Ala]. The incomplete restoration of IGF2 RNA splicing and translation by IMP1[Ser181Ala] was reflected in the production of immunoreactive IGF2 polypeptide (Fig. 5D) and in the MEF proliferative response (Fig. 5E); after doxycycline induction of IMP1 expression, comparable numbers of cells were replated, and the cell number was determined over the next $3 \mathrm{~d}$ in the presence of doxycycline. The cells expressing IMP1 wild type and IMP1[Ser181Asp] proliferated at similar rates, more than doubling in number over this interval (as do wild-type MEFs), whereas cells expressing IMP1[Ser181Ala] proliferated at $\sim 50 \%$ the rate of the other IMP1 variants, behavior that parallels the ability of these IMP1 variants to restore IGF2 expression. These results indicate that IMP[Ser181] phosphorylation is critical to the ability of IMP1 to enable IGF2 mRNA splicing and especially the initiation of L3IGF2 mRNA translation.

\section{IMP1 phosphorylation is critical for IMP1 binding} to the IGF2-L3 5' UTR

The selective effect of IMP1 on L3-mediated translation was explored further by examining the effect of IMP1 depletion (Fig. 6A) and overexpression (Fig. 6B) on the translational efficiency of L3-Luc and L4-Luc mRNAs during transient expression in RD rhabdomyosarcoma cells. Depletion of endogenous IMPs with shRNA (IMP1) reduced the translation of L3-Luc by $80 \%$ (IMP1) with minimal effect on L4-Luc expression (Fig. 6A). Conversely, Tet-induced overexpression of IMP1 approximately twofold over endogenous levels increases expression of L3-Luc approximately twofold with no significant alteration of L4-Luc expression (Fig. 6B). In contrast, comparable overexpression IMP1[Ser181Ala] increased L3-Luc expression only 1.2-fold (Fig. 6C, top). Whereas the amount of L3-Luc mRNA recovered with IMP1[Ser181Ala] was reduced by $80 \%$ as compared with IMP1 wild type, the amount of L3-Luc mRNA retrieved with IMP1[Ser181Asp] was very similar to that recovered with IMP1 wild type (Fig. 6C, bottom). Thus, it is likely that the lesser ability of IMP1[Ser181Ala] to promote IGF2-L3 mRNA translation is largely attributable to its lesser ability to bind the IGF2-L3 5' UTR. 


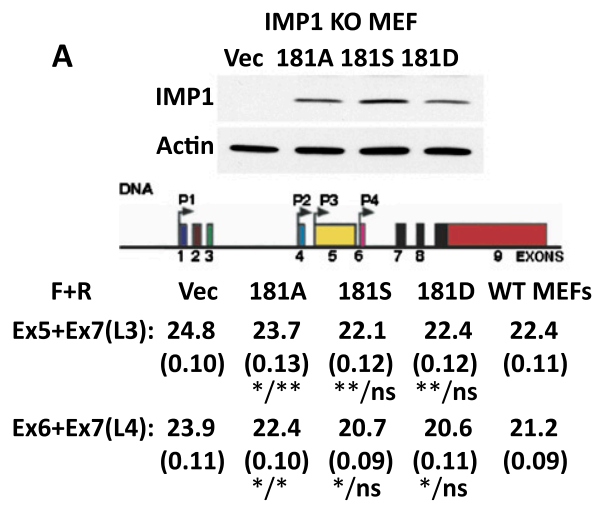

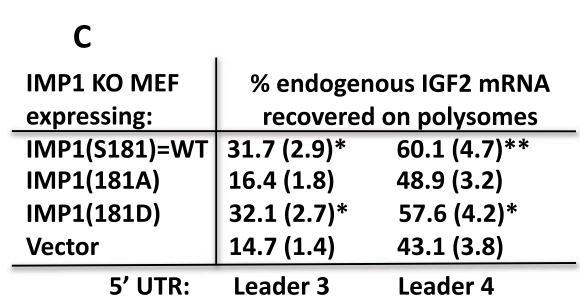

\section{D}

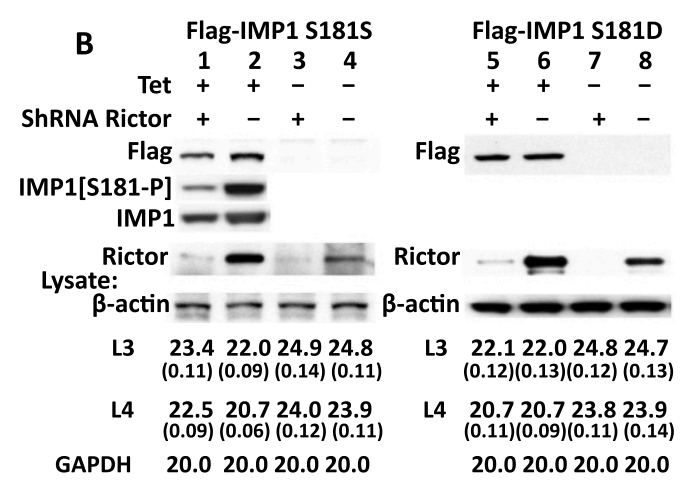

IGF2 ng protein: WT KO +181S +181A +181D +vec

$\begin{array}{lllll}\text { in medium (SD) } & \text { (27) }(13) \quad(26) \quad(12) & \text { (31) } & \text { (13) }\end{array}$

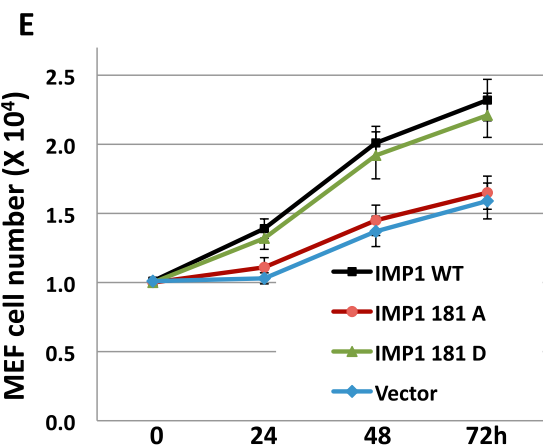

Figure 5. mTORC2-catalyzed IMP1(Ser181) phosphorylation is required to enable IMP1 regulation of IGF2 mRNA splicing and translation in MEFs. (A) Stable expression of IMP1 wild type (WT) or IMP1(Ser181D) but not IMP1(Ser181A) restores IGF2 RNA splicing in IMP1-null MEFs. The blot shows the level of IMP1 variant polypeptides in the stably transformed IMP1-null MEF lines. A cartoon depicting the exon-intron structure of the IGF2 gene is shown. Shown below the cartoon are the Ct values ( \pm SD) from qPCR assays reflecting the abundance of spliced mRNAs encoding L3-IGF2 (Ex5 + Ex7) and L4-IGF2 (Ex6 + Ex7) mRNAs in total RNA extracted from each of the four MEF lines shown in the blot. $\left(^{\star}\right) P<0.05$; $\left(^{\star \star}\right) P<0.01$; left of slash, versus Vec; right of slash, versus wild type. $(B)$ Rictor depletion impairs the ability of IMP1 wild type but not IMP1(Ser181Asp) to rescue L3 mRNA and L4 mRNA expression. IMP1-null MEF lines stably transformed with tetracycline-inducible IMP1 wild type or IMP1(Ser181Asp) cDNAs were infected with control lentivirus or lentivirus encoding rictor shRNA and selected with puromycin $(2 \mu \mathrm{g} / \mathrm{mL})$. Forty-eight hours later, cells were treated with doxycycline $(1 \mu \mathrm{g} / \mathrm{mL})$ or carrier and harvested $1 \mathrm{~d}$ later. Extracts were subjected to immunoblot for endogenous rictor, Flag-IMP1, $\beta$-actin, and, where relevant, IMP1(Ser181P). Shown below the blots are the Ct values $( \pm$ SD) from qPCR assays reflecting the abundance of the mRNAs encoding GAPDH, L3-IGF2, and L4-IGF2 mRNAs in total RNA. The value in lane 1 differs from that in lanes 2 and 3, and lane 2 differs from lane 4 , all $P<0.01$; lanes 5 and 6 do not differ from each other, but both differ from lanes 7 and $8, P<$ 0.01. (C) Stable expression of IMP1 wild type or IMP1(Ser181D) but not IMP1(Ser181A) restores L3-IGF2 mRNA translation in IMP1null MEFs. The percentage ( \pm SD) of total L4-IGF2 mRNA and L3-IGF2 mRNAs in the polysomal fractions of sucrose density gradients prepared from extracts of the IMP1-null MEFs stably expressing the recombinant IMP1 variants shown in $A .\left(^{\star}\right) P<0.05 ;\left(^{\star \star}\right) P<0.01$ versus vector. $(D)$ Stable expression of IMP1 wild type or IMP1(Ser181D) but not IMP1(Ser181A) restores IGF2 polypeptide production by IMP1-null MEFs to wild-type levels. Stably transformed IMP1-null MEFs expressing recombinant IMP1 variants shown in $A$ were grown in the presence of doxycycline. The level of the IMP1 variants induced by doxycycline is shown in $A$. Equal numbers of each set of MEFs were replated in the presence of doxycycline. After $3 \mathrm{~d}$, the medium was harvested, concentrated, and assayed for IGF2 ( \pm SD) as described in the Materials and Methods. $\left(^{\star}\right) P<0.05$; left of slash, versus wild type; right of slash, versus Vec. (E) Stable expression of IMP1 wild type or IMP1(Ser181D) but not IMP1(Ser181A) restores the proliferation of IMP1-null MEFs to wild-type levels. Stably transformed IMP1-null MEFs expressing recombinant IMP1 variants shown in $A$ were grown in the presence of doxycycline. The level of the IMP1 variants induced by doxycycline is shown in $A$. Equal numbers of each set of MEFs were replated in the presence of doxycycline at day 0 , and cell counts $( \pm$ SD) measured daily are shown. The cell counts for IMP1 wild type and IMP1(Ser181Asp) are higher than vector (IMP1 KO) and IMP1(Ser181Ala) at $24 \mathrm{~h}$ and thereafter $(P<0.05)$, whereas IMP1(Ser181Ala) and vector are not different.

IMP1 is absolutely required for translation of IGF2-L3 $m R N A$ in a reticulocyte lysate

We next examined the effects of IMP1 variants on the translational initiation of L3-luciferase and L4-luciferase mRNAs in vitro in rabbit reticulocyte lysates (Fig. 7A). Consistent with previous observations (DeMoor et al. 1994; Dai et al. 2011), luciferase mRNA bearing an
L4-IGF2 5' UTR is translated effectively by reticulocyte lysates, as shown by the appearance of luciferase activity; however, luciferase mRNA bearing the L3-IGF2 5' UTR shows essentially no translation. Addition of increasing amounts of recombinant wild-type IMP1 causes a modest dose-dependent inhibition of the L4-IGF2-luciferase translation, plateauing at $\sim 50 \%$ inhibition. In contrast, addition of recombinant wild-type IMP1 stimulates the 
translation of L3-IGF2-luciferase in a dose-dependent manner; in the presence of maximal IMP1, the luciferase activity increases from background levels at least 40-fold over baseline and to a level equivalent to that observed when L4-IGF2-luciferase is translated in the presence of IMP1. The IMP1 variant [Ser181Asp] is nearly as effective as wild-type IMP1 in stimulating L3-IGF2-luciferase mRNA translation, whereas IMP1[Ser181Ala] is only $20 \%$ as effective as wild type (Fig. 7A), consistent with the relative ability of IMP1[Ser181Ala] as compared with wildtype IMP1 to bind L3-IGF2 mRNA and promote L3-IGF2Luc translation during transient expression in RD cells.

Previous work has established that L3-IGF2 mRNA is translated in mammalian cells by cap-independent internal ribosome entry (Dai et al. 2011); to determine whether this is so in the reticulocyte lysate, we compared $\beta$-globin-luciferase and L3-IGF2-luciferase translation from 5'-capped and uncapped mRNAs (Fig. 7B); whereas an unmethylated $5^{\prime}$ cap results in greatly diminished expression of the $\beta$-globin-Luc mRNA in the presence (or absence) of IMP1, omission of the $5^{\prime}$ cap has little effect on the IMP1-stimulated translation of L3-IGF2-Luc. Thus, as in the cell, IMP-stimulated IGF2-L3-luciferase translational initiation occurs by cap-independent internal ribosomal entry.

\section{Discussion}

IGF2 and IGF1 are the major determinants of birth size in mice through their ability to promote overall cellular

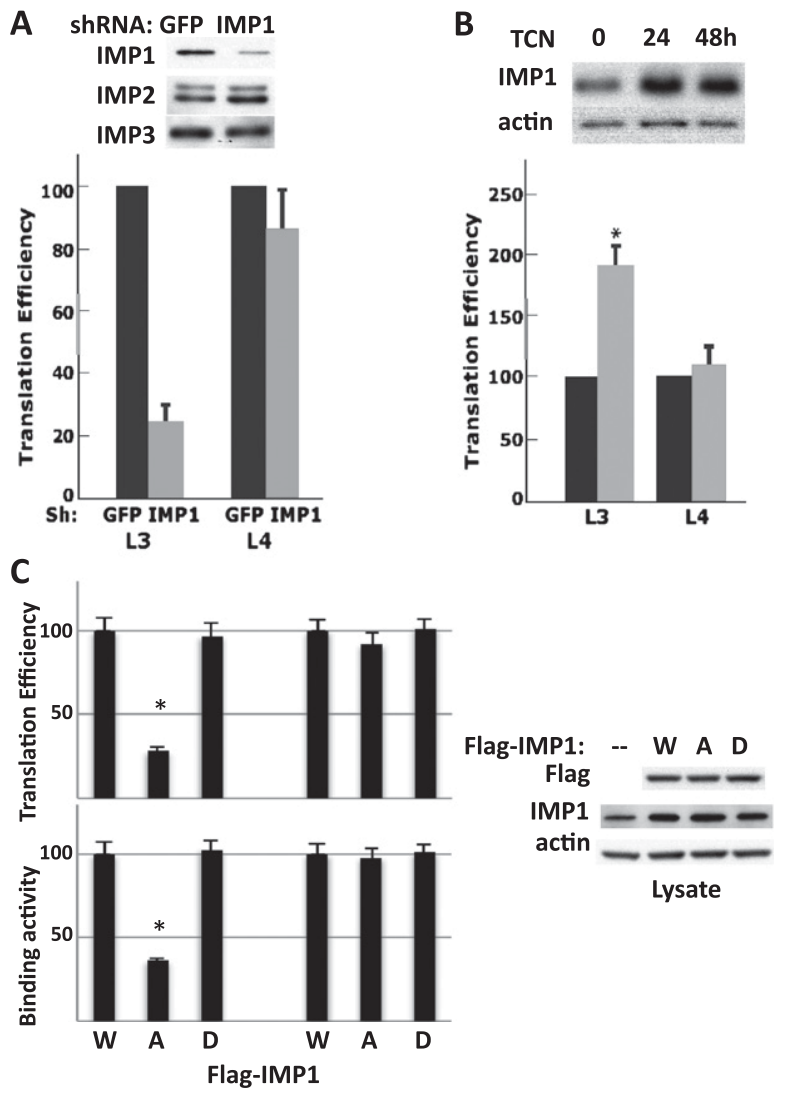

proliferation during the second half of embryonic growth. Lack of IGF2 (or IGF1) reduces birth size by $40 \%$ as compared with wild type (Efstratiadis 1998), and lack of the IGF2 RNA-binding protein IMP1 reduces birth size by $20 \%-25 \%$, despite continued expression of the homologous IMP2 and IMP3 (Hansen et al. 2004). MEFs that lack IMP1 polypeptide expression or that express an IMP1 polypeptide that cannot be phosphorylated at Ser181 exhibit an $80 \%$ decreased production of IGF2. Such MEFs proliferate at $\sim 50 \%$ the rate of wild type, a proliferative defect that is rescued by exogenous IGF2. Although overall IGF2 RNA levels are unaltered in the IMP1-null MEFs as compared with wild type, lack of IMP1 disturbs IGF2 RNA post-transcriptional processing at several

Figure 6. IMP1(Ser181-P) phosphorylation promotes IMP1 binding to the IGF2-L3 5' UTR and promotes L3-mediated translation in RD cells. (A) Depletion of IMP1 with shRNA from $\mathrm{RD}$ cells selectively impairs the translational efficiency of L3-luciferase. Plasmids encoding IGF2-L3 firefly luciferase or IGF2-L4 firefly luciferase, each together with a plasmid encoding Renilla luciferase, were transfected into RD cells stably expressing shRNAs directed against GFP (black bars) or IMP1 (gray bars), and the cells were harvested $48 \mathrm{~h}$ later. Extracts were assayed for firefly and Renilla luciferase activity and by qPCR for the content of firefly luciferase mRNA. The activity of firefly luciferase was divided by the activity of Renilla luciferase to give a normalized firefly luciferase activity; "translational efficiency" was calculated by dividing the normalized firefly luciferase activity by the measured content of firefly luciferase mRNA, setting to 100 the value of this dividend for the L3-Luc and L4-Luc conditions in the cells expressing GFP-shRNA. Each experiment was performed in triplicate, and the combined results of three experiments is shown \pm SEM. $(B)$ Overexpression of IMP1 in RD cells selectively enhances the translational efficiency of L3-luciferase. Plasmids encoding the 5' UTRs of IGF2-L3 or IGF2-L4 fused to firefly luciferase, each together with a plasmid encoding Renilla luciferase, were transfected into RD cells that stably express recombinant IMP1 in a doxycycline-inducible manner. The cells were treated with doxycycline (gray bars) or carrier (black bars) for $24 \mathrm{~h}$. "Translational efficiency" was calculated as in $A$. Each experiment was performed in triplicate, and the combined results of three experiments is shown \pm SEM. (C) IMP1(Ser181Ala) binds poorly to the IGF2-L3 $5^{\prime}$ UTR and is defective in promoting the translational efficiency of L3-luciferase. RD cells stably expressing doxycycline-inducible Flag-IMP1 wild type (WT), Flag-IMP1(Ser181Ala), or Flag-IMP1(Ser181Asp) were treated with doxycycline to increase total IMP1 levels about twofold over endogenous. Flag-IMP1 expression increases the translational efficiency of IGF2-L3-luciferase about twofold but does not alter IGF2-L4-luciferase translation, as in $B$. The relative effect of the Flag-IMP1 variants on L3-luciferase and L4luciferase translational efficiency (top graph) and on their binding of luciferase mRNA as a fraction of total luciferase mRNA ([W] 100; bottom graph) was measured in triplicate in three experiments. Results shown are \pm SEM; the values for IMP1(Ser181 Ala) are different from wild type and Ser181Asp; (*) $P<0.05$. The immunoblot illustrates the extent of doxycycline-induced expression of the Flag-IMP1 variants in relation to the level of endogenous IMP1. 
A

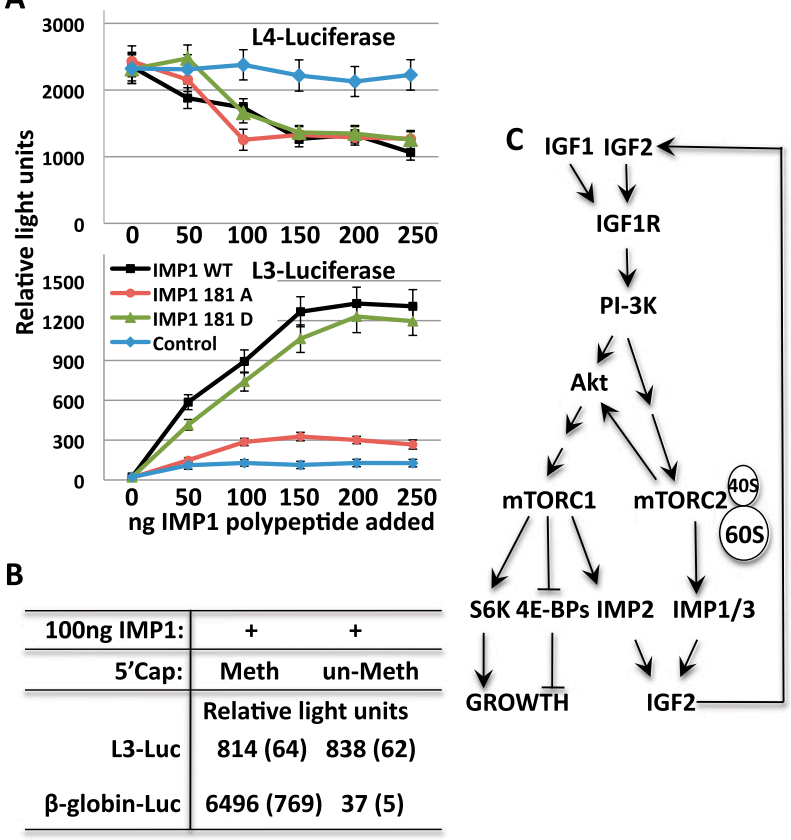

Figure 7. IMP1(Ser181) phosphorylation is required for translation of IGF2-L3-mediated translational initiation in vitro by internal ribosomal entry. (A) Translation of IGF2-L3-luciferase mRNA in rabbit reticulocyte lysates is increased from background levels by $>40$-fold by IMP1 wild type (WT) or IMP1(Ser181D) but not IMP1(Ser181A). (RLU) Results of triplicate measurements \pm SD in three separate experiments. For L4-luciferase, all IMP variants, starting at $100 \mathrm{ng}$, yield RLU values less than control; $P<0.05$. For L3-luciferase, all amounts of IMP1 wild type and Ser181Asp give RLU values greater than control $(P<0.05)$, whereas RLU values for IMP1(Ser181Ala) are greater than control starting at $100 \mathrm{ng}(P<0.05)$. (B) IMP1-stimulated IGF2-L3luciferase mRNA translation in a rabbit reticulocyte lysate is insensitive to $5^{\prime}$ cap methylation, whereas translation of $\beta$-globinluciferase mRNA is abolished by omission of $5^{\prime}$ cap methylation. (C) mTORC2 controls the IGF2 production and proliferation of MEFs. mTORC2-catalyzed, cotranslational phosphorylation of IMP1 (and IMP3) is necessary for IGF2 RNA processing and polypeptide production and thus normal mouse embryonic growth.

sites; thus, the levels of the most abundant spliced mature IGF2 mRNAs, which encode the 5' UTRs known as L3 and L4, are reduced by $\sim 80 \%$, and translational initiation of the L3-IGF2 mRNA is reduced by $\sim 50 \%$. The IMP1[Ser181Ala] polypeptide has minimal ability to rescue these defects; IGF2-L3 and IGF2-L4 mRNA levels remain low, and the translation of L3-IGF2 is not significantly enhanced by the nonphosphorylated IMP1.

The present finding that IMP1[Ser181] phosphorylation, so critical to IGF2 expression, is catalyzed by the mTOR kinase uncovers another mechanism by which mTOR regulates organismal size (Fig. 7C). Surprisingly, however, this growth regulatory phosphorylation is mediated by mTORC2 rather than mTORC1 and is a constitutive cotranslational rather than a regulated post-translational modification. This finding was unexpected, inasmuch as we previously observed that the dual phosphorylation of
IMP2[Ser162/164], which also promotes the ability of IMP2 to enhance L3-IGF2 mRNA translation, is catalyzed in a nutrient-dependent manner by mTORC1 (Dai et al. 2011), as are nearly all actions of mTOR on growth regulation previously described; e.g., the phosphorylation of 4E-BP, TIF-IA, Maf1, S6K1, and its substrates (Hietakangas and Cohen 2009). This dramatic difference in the mTOR regulation of IMP1 and IMP2 may perhaps be related to the differential expression of these RNAbinding proteins; whereas both are expressed during embryogenesis in mice, IMP1 expression, like that of IGF2, is largely extinguished before birth, whereas IMP2 expression persists postnatally and is widespread (Nielsen et al 1999; Dai et al. 2011). Thus, IMP1 may serve a relatively specific role in the mouse embryo as a predominant support of IGF2 expression (perhaps with IMP3) and thus cell proliferation, whereas IMP2, when expressed in adult mouse tissues, must bind a different cohort of RNAs, some of whose physiological functions are subject to insulin/nutrient regulation. It should be emphasized that although a large number of candidate IMP1- and IMP2-associated RNAs have been identified (Hafner et al. 2010), which of these RNAs are physiologically relevant partners in vivo, apart from IGF2 and a few others (e.g., $\beta$-actin and c-myc) (Yisraeli 2005), is uncertain. Moreover, it remains to be determined whether IMP1[Ser181] phosphorylation is important for IMP1 binding to other partners; e.g., the IGF2-mRNA or $\beta$-actinmRNA 3' UTRs or the c-myc-coding region.

In addition to IMP1's previously appreciated role in IGF2 mRNA translation, the present results uncover an important role for IMP1 in IGF2 RNA splicing. Although IMP1 has been identified as a component of the RNA spliceosome (Herold et al. 2009), its specific contributions to IGF2 RNA splicing, and thus the role of [Ser181] phosphorylation, are not known. Nevertheless, the deficient splicing of both IGF2-L3 and IGF2-L4 mRNAs in IMP1-null MEFs suggests that IMP1 interacts at multiple sites on the IGF2 RNA transcript. In regard to IGF2 mRNA translation, loss of cotranslational [Ser181] phosphorylation markedly impairs the ability of IMP1 to bind the IGF2-L3 5' UTR and thereby configure an L3 RNA structure capable of supporting internal ribosomal entry. It is likely that the phosphorylation of homologous IMP3 site [Ser183] subserves a similar function. The mechanism by which the binding of IMP1 enables L3 translation is of considerable interest (Nielsen et al. 1995; Ikenoue et al. 2008; Dai et al. 2011). The availability of IMP1-null MEFs, together with the ability of IMP1 to enable L3 RNA translation by internal ribosomal entry in a rabbit reticulocyte lysate in an "all-or-none" manner, should enable the determination of what other proteins, if any, are needed to enable ribosome binding to L3.

The finding that IMP1 phosphorylation by mTORC2 occurs cotranslationally, together with the recent demonstration that TORC2 catalyzes the cotranslational phosphorylation of the "turn" motif of several AGC kinases, suggests that cotranslational phosphorylation may be a core function of TORC2 (Oh et al. 2010). This possibility is supported by the earlier finding that TORC2 
associates specifically with the ribosome through the $60 \mathrm{~S}$ subunit, with rictor binding to L23a and L26, proteins situated near the polypeptide exit site (Oh et al. 2010). Association with the ribosome appears to be capable of activating mTORC2, independent of ongoing protein synthesis (Zinzalla et al. 2011). It will be of interest to define the overall scope of cotranslational phosphorylation, the contribution of $\mathrm{mTORC} 2$ to this process, and its functional consequences.

\section{Materials and methods}

\section{MEF preparation and culture}

MEFs were generated from embryos isolated at E13.5 produced by the mating of IMP1 heterozygotes. Embryos were genotyped, and MEFs were cultured in Dulbecco's modified Eagle's medium (DMEM) supplemented with $10 \%$ FBS at $37^{\circ} \mathrm{C}$ in $5 \% \mathrm{CO}_{2}$. The human RD cells (American Type Culture Collection, CCL-136) $293 \mathrm{~T}$ and 293E were cultured under the same conditions.

\section{Antibody generation}

Polyclonal antisera to IMP1 and IMP3 were generated by immunization of rabbits with the synthetic peptides CEKVFAEHKISYamide and CESIFKDAKIPVamide, respectively, coupled to KLH by their N-terminal cysteine. Antibodies were purified by peptide affinity chromatography. Antibodies reactive with IMP1[Ser181-PO4] and IMP3[Ser183-PO4] were elicited by immunization of rabbits with CQPRQGS(PO4)PVAAGamide and CSSRQGS(PO4)PGSVSamide, respectively, coupled to KLH. After depleting these sera of antibodies reactive with the corresponding nonphosphorylated peptide, phospho-specific antibodies were affinity-purified with the immunizing peptides. Immunizations were performed at Cocalico Biologicals, Inc.

\section{Mass spectrometry}

Identification of protein phosphorylation sites was performed at the Taplin Biological Mass Spectrometry Facility (Harvard Medical School); after SDS-PAGE and Coomassie staining, relevant bands were excised and subjected to tryptic digestion in situ, and extracellular peptides were separated and analyzed by microcapillary LC-MS/MS.

\section{$R N A$ isolation and $q R T-P C R$}

RNA was extracted with RNeasy kit (Qiagen) and precipitated with ethanol. PolyA ${ }^{+}$RNAs were isolated with immobilized oligo (dT). IGF2, GAPDH, and $\beta$-actin RNA levels were examined by qRT-PCR. The PCR primers were designed using Primer3 software (Massachusetts Institute of Technology) and synthesized by MGH DNA core. Each sample was run in triplicate. The experiment was performed at least three times independently. The primers used were IGF2 exon4for (TCTGAAGCCACAGAAATTAGAGG), IGF2 exon4-rev (GGGGAGCTTAATTTTAATTGCT), IGF2 exon5-for (TCTAT CTACCTCAACACCCCATT), IGF2 exon5-rev (CGACGGAG CCCTCAGCCGTCGT), IGF2 exon7-for (GGGGATCCCAGT GGGGAAGT), IGF2 exon7-rev (GAAGTAGAAGCCGCGG TCCGAACA), IGF2 exon4,5' -for (TCCGATCCTCCTGCGCC ACGGACC) to IGF2 exon7,3'-rev (GAAGTAGAAGCCGCG GTCCGAACA), IGF2 exon5, 5' -for (TGTTCGGTTTGCAT ACCCGCAGCAGG) to IGF2 exon7,3'-rev (GAAGTAGAAG
CCGCGGTCCGAACA) IGF2 exon6,5'-for (ACATTAGCTT CTCCTGTGAGAACC) to IGF2 exon7, $3^{\prime}$-rev (GAAGTAGA AGCCGCGGTCCGAACA), GAPDH-for (GAGTATGTCGT GGAGTCTACTGG), and GAPDH-rev (TCATGAGCCCTTCC ACAATGCC).

\section{Messenger ribonucleoprotein immunoprecipitation assay}

Exponentially growing human RD cells or MEF cells were washed with phosphate-buffered saline (PBS) at $4^{\circ} \mathrm{C}$ and lysed by incubation for $10 \mathrm{~min}$ on ice with a lysis buffer $140 \mathrm{mM} \mathrm{KCl}$, $1.5 \mathrm{mM} \mathrm{MgCl} 2,20 \mathrm{mM}$ Tris- $\mathrm{HCl}$ at $\mathrm{pH} 7.4,0.5 \%$ nonidet $\mathrm{P}-40$, $0.5 \mathrm{mM}$ dithiothreitol, $1 \mathrm{U} / \mu \mathrm{L}$ RNase inhibitor, one complete EDTA-free protease inhibitor cocktail tablet). All subsequent steps were at $4^{\circ} \mathrm{C}$. The lysate was centrifuged at $12,000 \mathrm{rpm}$ for $10 \mathrm{~min}$, and the supernatant was transferred to a fresh $1.5-\mathrm{mL}$ tube. Total protein concentration in the lysates was measured by Bradford assay. For immunoprecipitation, cytoplasmic lysate proteins were incubated with Flag agarose beads or protein A Dyna magnetic beads precoated with rabbit IgG or IMP1 antibody for $2 \mathrm{~h}$ at $4^{\circ} \mathrm{C}$ with rotation. Beads were extensively washed with lysis buffer and digested with DNase I (Roche Applied Science) and protease K (Sigma). RNA was extracted with RNeasy kit (Qiagen) and precipitated with ethanol. Real-time PCR was performed to examine RNAs associated with cytoplasmic IMP2 as described above.

\section{Sucrose density gradient centrifugation}

Exponentially growing MEFs were collected and washed in icecold PBS containing $10 \mu \mathrm{g} / \mathrm{mL}$ cycloheximide. The cell pellet was resuspended in $500 \mu \mathrm{L}$ of lysis buffer as described above and centrifuged at $10,000 \mathrm{~g}$ for $10 \mathrm{~min}$. The supernatant was applied to a linear $20 \%-47 \%$ sucrose gradient in $20 \mathrm{mM}$ Tris-HCL $(\mathrm{pH}$ 7.5), $140 \mathrm{mM} \mathrm{KCl}$, and $5 \mathrm{mM} \mathrm{MgCl}_{2}$ and centrifuged at 40,000 $\mathrm{rpm}$ for $3 \mathrm{~h}$ with Beckman SW41 rotors. Fractions of $1 \mathrm{~mL}$ were collected with concomitant measurement of the absorbance at $260 \mathrm{~nm}$, followed by phenol/chloroform extraction. Real-time PCR was performed to examine the abundance of RNAs in different fractions.

\section{Generation of tetracycline-inducible MEF cell lines}

The IMP1-null MEFs that stably overexpress Flag-IMP1 variants were generated using pcDNA6/TR and pcDNA5/TO vectors according to the manufacturer's instructions (Invitrogen). The mutants of IMP1 were created using the QuikChange sitedirected mutagenesis kit (Stratagene).

\section{In vitro translation}

In vitro translation was performed using Flexi Rabbit Reticulocyte lysate system (Promega) according to the manufacturer's instructions. Reactions were started by addition of the in vitro transcribed, purified RNAs encoding IGF2-L3 or IGF2-L4 fused $5^{\prime}$ to the luciferase coding sequence and were carried out for $1 \mathrm{~h}$ at $37^{\circ} \mathrm{C}$. IMP1 polypeptides were generated in $293 \mathrm{~T}$ cells transfected with vectors encoding Flag-IMP1 variants or with empty vector, lysates were incubated with agarose-immobilized anti-Flag antibody, and beads were washed and eluted with Flag peptide. The eluted IMP1 variants were added to the reaction prior to addition of RNA; the Flag eluate from the beads incubated with lysate from vector transfected cells served as control. The dual-luciferase reporter assay (Promega) was used for detection of the luciferase polypeptide translated in vitro. 


\section{Coupled transcription/translation and kinase assay}

In vitro transcription/translation was performed using the TNT T7 Quick-Coupled Transcription/Translation system (Promega) containing an IMP1 DNA template according to the manufacturer's instructions at $37^{\circ} \mathrm{C}$. In some reactions, Flag-mTOR immunopurified from HEK293T cell lysates was added to the transcription/translation reaction before initiation of translation, in which case, SDS sample buffer was added $2 \mathrm{~h}$ later. Alternatively, Flag-mTOR was added after translation had proceeded for $2 \mathrm{~h}$ and was terminated by addition of puromycin to displace nascent polypeptides from the ribosomes; in this mode, incubation was continued for an additional $2 \mathrm{~h}$ followed by addition of SDS sample buffer. Reactions were analyzed by Western Blot after SDS-PAGE.

\section{Cell counts}

Equal numbers of MEFs were plated and maintained for 24-72 h. Cells were stained with Trypan blue according to the manufacturer's protocol. Only cells with unstained nuclei were counted.

\section{Measurement of IGF2 concentration}

The concentration of secreted IGF2 in culture medium was determined by Western blot after concentration by the Amicon Ultra-15 centrifugal filter units (Millipore) according to the manufacturer's instruction. A standard curve was prepared using human recombinant IGF2, and samples were immunoblotted after SDS-PAGE. The IGF2 concentration in the medium samples was interpolated from the standard curve using ImageJ software.

\section{Creation of shRNA-containing lentiviral particles}

shRNA GFP-mTOR (pLL3.7), Rictor, Raptor, and GFP constructs were constructed in the pLKO1 backbone. The plasmid, along with a packaging and envelop plasmid (Addgene), was transfected into HEK293T cells using Lipofectamine (Invitrogen) according to the manufacturer's instructions. After $24 \mathrm{~h}$, the medium was changed (20 mM HEPES, 30\% FBS/DMEM, 2 mM sodium butyrate). After $24 \mathrm{~h}$, the medium was collected, filtered through $0.45-\mu \mathrm{m}$ filters, and flash-frozen in liquid nitrogen. Two mTOR shRNA were constructed in pLL3.7 using the sequences AATGTTGACCAATGCTATGGA from human and rat mTOR and CGAAATTTTGGACGGTGTGGAA from human mTOR. Both give robust depletion of $\mathrm{mTOR}$ in human cell lines.

\section{Lentiviral delivery of shRNA}

Human RD cells were infected with lentiviral particles. Clones were picked from cell pools selected via puromycin and were tested for knockdown efficiency via Western blot. The shRNA GFP served as a negative control.

Other reagents were obtained from the following sources: Antibodies to phospho-Thr36/47 4E-BP1, 4E-BP1, phosphoThr389-S6K were from Cell Signaling Technology; total S6K was from Santa Cruz Biotechnology; IGF2 and SC35 were from Abcam; SF1 was from Millipore; puromycin antibody was from KeraFast; actin was from Sigma; mTOR, Raptor, and Rictor were as in Oshiro et al. (2007); and anti-mouse, anti-rabbit, and antigoat secondary antibodies were from Santa Cruz Biotechnology.

IMP1 shRNA, Flag-M2 antibody, Flag-M2-agarose, human recombinant IGF2, NaF, SP600125, staurosporine, kenpaullone, and insulin were from Sigma-Aldrich. DNase, RNases, RNase inhibitor, and Complete Protease Mixture were from Roche
Applied Science; DMEM, inactivated fetal calf serum, puromycin, Dynabeads protein A, Lipofectamine, and Lipofectamine 2000 were from Invitrogen. PD184352 and SB203580 were from Axon Medchem. Calyculin A was from LC Laboratories. Torin 1 was from Tocris bioscience.

\section{Acknowledgments}

We thank E. Jacinto and colleagues for advice regarding the analysis of cotranslational phosphorylation. The work was supported by NIH grants DK17776, CA73818, and DK057521 (to J.A.) and by institutional funds.

\section{References}

Bain J, McLauchlan H, Elliott M, Cohen P. 2003. The specificities of protein kinase inhibitors: An update. Biochem J 371: 199-204.

Constância $M$, Hemberger $M$, Hughes $J$, Dean W, FergusonSmith A, Fundele R, Stewart F, Kelsey G, Fowden A, Sibley C, et al. 2002. Placental-specific IGF-II is a major modulator of placental and fetal growth. Nature 417: 945-948.

Dai N, Rapley J, Angel M, Yanik MF, Blower MD, Avruch J. 2011. mTOR phosphorylates IMP2 to promote IGF2 mRNA translation by internal ribosomal entry. Genes Dev 25: 11591172.

Davies SP, Reddy H, Caivano M, Cohen P. 2000. Specificity and mechanism of action of some commonly used protein kinase inhibitors. Biochem J 351: 95-105.

DeChiara TM, Efstratiadis A, Robertson EJ. 1990. A growthdeficiency phenotype in heterozygous mice carrying an insulin-like growth factor II gene disrupted by targeting. Nature 345: 78-80.

De Moor CH, Jansen M, Bonte E.J, Thomas AA, Sussenbach JS, Van den Brande JL. 1994. Influence of the four leader sequences of the human insulin-like-growth-factor- 2 mRNAs on the expression of reporter genes. Eur I Biochem 226: 10391047.

Efstratiadis A. 1998. Genetics of mouse growth. Int I Dev Biol 42: 955-976.

Favre B, Turowski P, Hemmings BA. 1997. Differential inhibition and posttranslational modification of protein phosphatase 1 and 2A in MCF7 cells treated with calyculin-A, okadaic acid, and tautomycin. I Biol Chem 272: 1385613863.

Hafner M, Landthaler M, Burger L, Khorshid M, Hausser J, Berninger $\mathrm{P}$, Rothballer A, Ascano M Jr, Jungkamp AC, Munschauer M, et al. 2010. Transcriptome-wide identification of RNA-binding protein and microRNA target sites by PAR-CLIP. Cell 141:129-141.

Hansen TV, Hammer NA, Nielsen J, Madsen M, Dalbaeck C, Wewer UM, Christiansen J, Nielsen FC. 2004. Dwarfism and impaired gut development in insulin-like growth factor II mRNA-binding protein 1-deficient mice. Mol Cell Biol 24: 4448-4464.

Herold N, Will CL, Wolf E, Kastner B, Urlaub H, Lührmann R. 2009. Conservation of the protein composition and electron microscopy structure of Drosophila melanogaster and human spliceosomal complexes. Mol Cell Biol 29: 281301.

Hietakangas V, Cohen SM. 2009. Regulation of tissue growth through nutrient sensing. Annu Rev Genet 43: 389-410.

Hüttelmaier S, Zenklusen D, Lederer M, Dictenberg J, Lorenz M, Meng X, Bassell GJ, Condeelis J, Singer RH. 2005. Spatial regulation of $\beta$-actin translation by Src-dependent phosphorylation of ZBP1. Nature 438: 512-515. 
Dai et al.

Huttlin EL, Jedrychowski MP, Elias JE, Goswami T, Rad R, Beausoleil SA, Villén J, Haas W, Sowa ME, Gygi SP. 2010. A tissue-specific atlas of mouse protein phosphorylation and expression. Cell 143: 1174-1189.

Ikenoue T, Inoki K, Yang Q, Zhou X, Guan KL. 2008. Essential function of TORC2 in PKC and Akt turn motif phosphorylation, maturation and signaling. EMBO J 27: 1919-1931.

Laplante M, Sabatini DM. 2012. mTOR signaling in growth control and disease. Cell 149: 27-93.

Lee JE, Pintar J, Efstratiadis A. 1990. Pattern of the insulin-like growth factor II gene expression during early mouse embryogenesis. Development 110: 151-159.

Newell S, Ward A, Graham C. 1994. Discriminating translation of insulin-like growth factor-II (IGF-II) during mouse embryogenesis. Mol Reprod Dev 39: 249-258.

Nielsen FC, Ostergaard L, Nielsen J, Christiansen J. 1995. Growthdependent translation of IGF-II mRNA by a rapamycinsensitive pathway. Nature 377:358-362.

Nielsen J, Christiansen J, Lykke-Andersen J, Johnsen AH, Wewer UM, Nielsen FC. 1999. A family of insulin-like growth factor II mRNA-binding proteins represses translation in late development. Mol Cell Biol 19: 1262-1270.

Nielsen FC, Nielsen J, Christiansen J. 2001. A family of IGF-II mRNA binding proteins (IMP) involved in RNA trafficking. Scand I Clin Lab Invest Suppl 234: 93-99.

Oh WJ, Wu CC, Kim SJ, Facchinetti V, Julien LA, Finlan M, Roux PP, Su B, Jacinto E. 2010. mTORC2 can associate with ribosomes to promote cotranslational phosphorylation and stability of nascent Akt polypeptide. EMBO I 29: 3939-3951.

Oshiro N, Takahashi R, Yoshino K, Tanimura K, Nakashima A, Eguchi S, Miyamoto T, Hara K, Takehana K, Avruch J, et al. 2007. The proline-rich Akt substrate of $40 \mathrm{kDa}$ (PRAS40) is a physiological substrate of mammalian target of rapamycin complex 1. J Biol Chem 282: 20329-20339.

Pestka S. 1971. Inhibitors of ribosome functions. Annu Rev Microbiol 25: 487-562.

Schmidt EK, Clavarino G, Ceppi M, Pierre P. 2009. SUnSET, a nonradioactive method to monitor protein synthesis. Nat Meth 6: 275-277.

Sussenbach JS. 1989. The gene structure of the insulin-like growth factor family. Prog Growth Factor Res 1: 33-48.

Thoreen CC, Kang SA, Chang JW, Liu Q, Zhang J, Gao Y, Reichling LJ, Sim T, Sabatini DM, Gray NS. 2009. An ATPcompetitive mammalian target of rapamycin inhibitor reveals rapamycin-resistant functions of mTORC1. I Biol Chem 28: 8023-8032.

Yisraeli JK. 2005. VICKZ proteins: A multi-talented family of regulatory RNA-binding proteins. Biol Cell 97: 87-96.

Zinzalla V, Stracka D, Oppliger W, Hall MN. 2011. Activation of mTORC2 by association with the ribosome. Cell 144: 757768. 


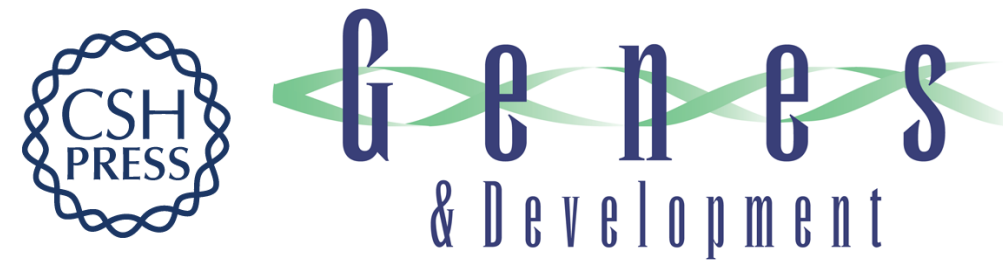

\section{mTOR complex 2 phosphorylates IMP1 cotranslationally to promote IGF2 production and the proliferation of mouse embryonic fibroblasts}

Ning Dai, Jan Christiansen, Finn C. Nielsen, et al.

Genes Dev. 2013, 27:

Access the most recent version at doi:10.1101/gad.209130.112

Supplemental
Material http://genesdev.cshlp.org/content/suppl/2013/02/06/27.3.301.DC1

References This article cites 29 articles, 7 of which can be accessed free at:

http://genesdev.cshlp.org/content/27/3/301.full.html\#ref-list-1

License

Email Alerting

Receive free email alerts when new articles cite this article - sign up in the box at the top

Service

right corner of the article or click here.

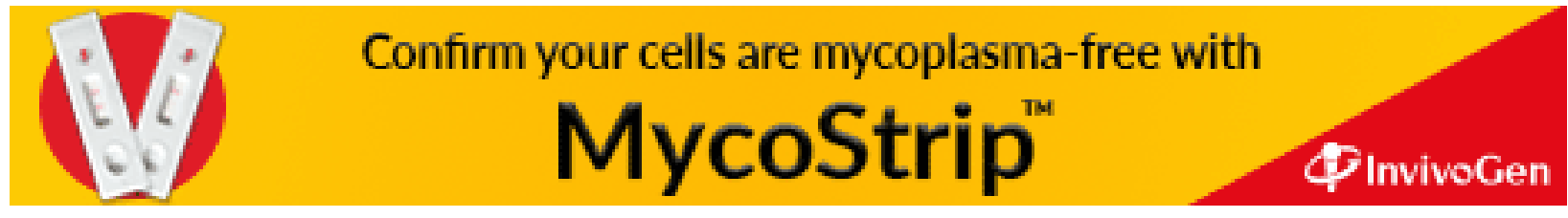

